\title{
Controlled terahertz frequency response and transparency of Josephson chains and superconducting multilayers
}

\author{
V. A. Yampol'skii, ${ }^{1,2}$ Sergey Savel'ev, ${ }^{1,3}$ O. V. Usatenko, ${ }^{2}$ S. S. Mel'nik, ${ }^{2}$ F. V. Kusmartsev, ${ }^{3}$ \\ A. A. Krokhin, ${ }^{4}$ and Franco Nori ${ }^{1,5}$ \\ ${ }^{1}$ Frontier Research System, The Institute of Physical and Chemical Research (RIKEN), Wako-shi, Saitama, 351-0198, Japan \\ ${ }^{2}$ A.Ya. Usikov Institute for Radiophysics and Electronics Ukrainian Academy of Sciences, 61085 Kharkov, Ukraine \\ ${ }^{3}$ Department of Physics, Loughborough University, Loughborough LE11 3TU, United Kingdom \\ ${ }^{4}$ Department of Physics, University of North Texas, Denton, Texas 76203, USA \\ ${ }^{5}$ Center for Theoretical Physics, Department of Physics, University of Michigan, Ann Arbor, Michigan 48109-1040, USA
}

(Received 16 July 2006; revised manuscript received 9 October 2006; published 26 January 2007)

\begin{abstract}
A fundamental property of wave propagation is Anderson localization, which affects the transfer of information, energy, mass, and charge in disordered media. This localization can manifest itself via, e.g., the metal-insulator transition. We exactly map the behavior of a quantum particle moving in a potential with correlated disorder to the sub-terahertz wave propagation in either Josephson chains or superconducting multilayers. When the Josephson junction parameters vary randomly, the sub- $\mathrm{THz}$ electromagnetic waves cannot propagate through these Josephson structures due to localization. For parameter variations with long-range correlations, we predict sharp transitions from transparent to reflective frequency regions for Josephson plasma waves. With appropriate choices of the correlation function, frequency windows with targeted or designed transparencies for $\mathrm{THz}$ or sub-THz electromagnetic waves could be achieved. This could be useful for tailoring the electromagnetic wave spectrum of Josephson arrays within the $\mathrm{THz}$ frequency range, which is important for applications in physics, astronomy, chemistry, biology, and medicine.
\end{abstract}

DOI: 10.1103/PhysRevB.75.014527

PACS number(s): 74.78.Fk, 74.50.+r

\section{INTRODUCTION}

The growing number of studies of Josephson structures is partly motivated by the sub-terahertz (up to several hundreds $\mathrm{GHz}$ ) frequency range of the electromagnetic waves (socalled Josephson plasma waves) propagating in these systems. Controlling Josephson plasma waves (JPWs) in Josephson arrays could be potentially useful for applications in physics, astronomy, chemistry, biology, and medicine, including THz imaging, spectroscopy, tomography, medical diagnosis, health monitoring, environmental control, as well as chemical and biological identification. ${ }^{1}$ These $\mathrm{THz}$ electromagnetic waves interact nonlinearly with the Josephson medium itself and with magnetic flux quanta (Josephson vortices), which, in turn, can be conveniently manipulated by varying an in-plane magnetic field and/or an out-of-plane electric current. Such an unprecedented level of controllability has been used to propose a set of well-integrated $\mathrm{THz}$ devices, ${ }^{2-6}$ including generators, tunable filters, detectors, wave mixers, lenses, converters, and amplifiers.

Furthermore, recent achievements in nanotechnology allow the fabrication of parallel-coupled one-dimensional (1D) Josephson junction chains ${ }^{7,8}$ [Fig. 1(a)], known as discrete Josephson transmission lines, and artificial superconducting multilayers $^{9}$ [Fig. 1(b)] with prescribed properties. For instance, one can controllably introduce some degree of disorder in these nanodevices. Since the theoretical models describing the Josephson arrays are well defined, it offers the opportunity of theoretically exploring and experimentally testing several fundamental concepts of low-dimensional physics and employing these for further developing superconducting $\mathrm{THz}$ devices.

Transport properties of disordered media have been stud- ied since the 1950s when Anderson localization, ${ }^{10,11}$ a fundamental concept for disordered systems, was proposed. For example, a white-noise (uncorrelated) disordered 1D chain exhibits insulating behavior at low temperatures due to mul-
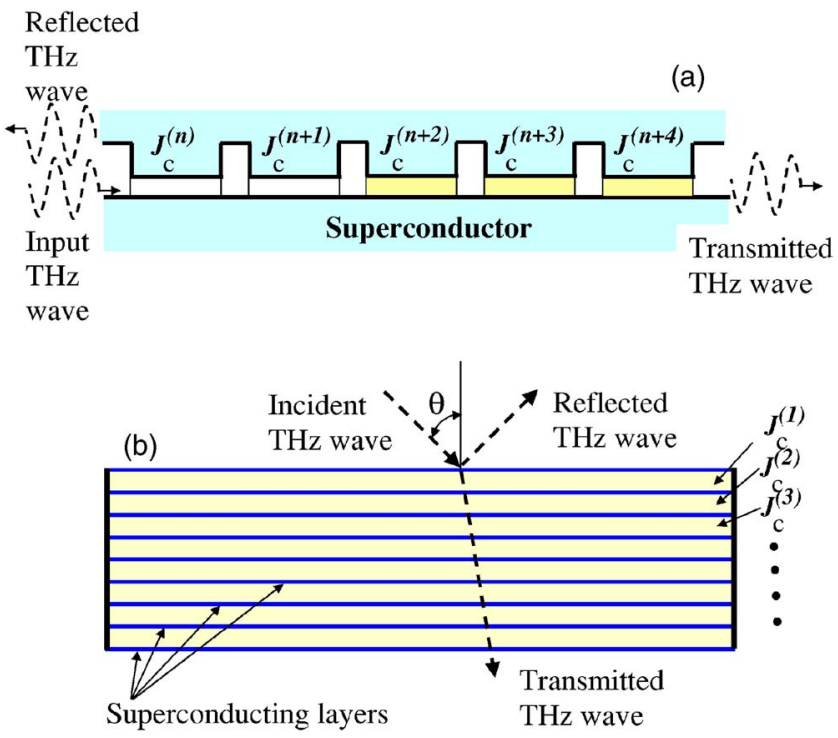

FIG. 1. (Color online) Schematic diagram of the parallelcoupled 1D Josephson junction array, known as discrete Josephson transmission line (a), and an artificial superconductingnonsuperconducting multilayer (b). For both systems, disorder can be introduced by varying the Josephson critical currents $J_{c}^{(n)}=J_{c}(1$ $\left.+\delta_{n}\right)=J_{c}(1 \pm \delta)$. These disordered Josephson transmission line (a) and artificial multilayers (b) can be mapped to a binary (dichotomic) chain with symbol " 1 " corresponding to $+\delta$ and " 0 " referring to $-\delta$. 
TABLE I. Comparison between localization of quantum particles and Josephson plasma waves. Here "tunability" refers to the ability to change the transport properties of a system by tuning an external parameter. This ability to tune and control transport could be useful for applications.

\begin{tabular}{l|c|c}
\hline \hline & Quantum particles & Josephson plasma waves \\
\hline Model & Tight-binding model & Linearized sine-Gordon model \\
\hline Mapping & $\begin{array}{c}\text { Energy } E \\
\text { quasi-momentum } k\end{array}$ & $\begin{array}{c}\text { Frequency } \omega \\
\text { Wave number } q\end{array}$ \\
\hline Randomness via & Random potential & Randomness in the Josephson junctions \\
\hline $\begin{array}{l}\text { Transparency edge } \\
\text { via correlations }\end{array}$ & Yes & $\begin{array}{c}\text { Yes, by changing either the incident angle } \\
\text { (for superconductors) or the frequency } \\
\text { (for the Josephson transmission line) }\end{array}$ \\
\hline How to observe & $\begin{array}{c}\text { E.g., metal-insulator transition, } \\
\text { transmission coefficient }\end{array}$ & $\begin{array}{c}\text { Transmissivity changes: from transparent to } \\
\text { opaque for JPW }\end{array}$ \\
\hline
\end{tabular}

Comparison beyond undamped, linear response

\begin{tabular}{l|c|c}
\hline Nonlinearity & $\begin{array}{c}\text { Can occur due to interparticle } \\
\text { interactions, but not easily controllable }\end{array}$ & $\begin{array}{c}\text { Controllable nonlinearity due to the nonlinear } J \\
\text { versus } \phi(J \propto \sin \phi)\end{array}$ \\
\hline Damping & Can occur, but not controllable & Controllable by changing temperature \\
\hline Tunability & No & Yes, by locally changing currents or fields \\
\hline \hline
\end{tabular}

tiple random scattering, i.e., as a result of destructive interference, a quantum particle turns out to be localized inside a finite length (calculated by Thouless ${ }^{12}$ ). In contrast, particles in a periodic (i.e., perfectly ordered) system can propagate long distances, giving rise to metal-like conducting behavior or peculiar properties of photonic crystals. ${ }^{13}$ Intermediate systems, described, for instance, by correlated random potentials (e.g., Refs. 14-16) or quasicrystalline structure (e.g., Refs. 17-19), can demonstrate either localization or delocalization, depending on their parameters. For instance, the degree of disorder drastically influences the particle mobilities. These phenomena involving disorder can be conveniently described using 1D tight-binding models (see, e.g., Refs. 16-18, 20, and 21).

For a long time, it was believed that localized and extended states in 1D potentials did not coexist and 1D systems could not display complex dynamic features such as the metal-insulator transition. An unexpected result was obtained in Refs. 22-24 that correlations can produce delocalized states in a random potential. Generalizing Thouless calculations, a relation between the pair correlation function and the localization length was derived in Refs. 14, 25, and 26. This relation shows that the correlation length can quickly change from infinite to a finite value at certain values of the particle energy, i.e., the localized and delocalized electron states do coexist in 1D random long-range-correlated systems. This means that the metal-insulator transition can be observed when the particle energy changes. This phenomenon, known as the mobility edge, is discussed, for instance, in Ref. 27. These findings have revised a previously accepted belief that any randomness (no matter how weak the randomness is) in 1D structures results in Anderson localization. ${ }^{11}$ In contrast, long-range (i.e., power law decaying) correlations can provide a continuum of extended states in the energy spectrum, and a metal-insulator transition.

Here we describe an analogy between the behavior of a quantum particle and the sub-terahertz wave propagation in 1D Josephson arrays and superconducting multilayers. For instance, it has already been shown ${ }^{4,28}$ that the frequency intervals where the electromagnetic JPWs can propagate form a band structure if a periodic superlattice of Josephson vortices is induced by an in-plane magnetic field in superconducting multilayers or a long 1D Josephson junction. This JPW photonic crystal, ${ }^{4}$ with gaps of forbidden frequency ranges tuned by the in-plane magnetic field, is a much more controllable analog of both the band structure in 1D conductors and standard photonic crystals. ${ }^{13}$ Here, we use this analogy (see Table I) between JPWs and quantum particles to show how to manipulate the $\mathrm{THz}$ and sub- $\mathrm{THz}$ electromagnetic wave localization and the transparency of Josephson chains and artificial superconducting multilayers via a controllable change of their disorder. In particular, we predict the frequency windows of transparency for samples with long-range correlated disorder. The effective random potential can be either continuously distributed or take a discrete number of values. We show that even a dichotomic (or binary) potential (i.e., discrete potential taking randomly only two possible values) can be used to construct transmission lines with any desirable transparency window (acoustic analogs of these have been studied in Ref. 16). Moreover, for 
superconducting multilayers, the transparency edge can also be observed as a function of either the incident angle or frequency of the sub-terahertz radiation, which could be useful for tailoring the electromagnetic wave spectrum of the array. The Josephson structures proposed here can be potentially useful for $\mathrm{THz}$ and sub-THz filters which are sensitive to either the frequency or the propagation direction of electromagnetic waves. The experimental realization of this proposal would be another example of controlled Anderson localization, among few, including experiments with GaAs$\mathrm{AlGaAs}$ random superlattices ${ }^{29}$ and with microwave transmission spectra through a single-mode waveguide with inserted correlated scatterers. ${ }^{30}$

\section{JOSEPHSON JUNCTION CHAINS}

\section{A. Designing a potential for JPWs in a quasi-1D Josephson array}

We consider JPWs propagating along the discrete Josephson transmission line schematically shown in Fig. 1(a). The gauge-invariant phase difference $\phi_{n}$, determining the electric and magnetic fields at the $n$th junction, obeys the discretized version of the perturbed sine-Gordon equation (see, e.g., Ref. 31):

$$
\frac{1}{\omega_{J}^{2}} \frac{d^{2} \phi_{n}}{d t^{2}}+\frac{1}{\omega_{R}} \frac{d \phi_{n}}{d t}+\frac{J_{c}^{(n)}}{J_{c}} \sin \phi_{n}=\frac{1}{a^{2}}\left(\phi_{n+1}+\phi_{n-1}-2 \phi_{n}\right)+j_{n} .
$$

Here, $\omega_{J}$ is the Josephson plasma frequency, $a=d / \lambda_{J}$ is the discreteness parameter, $d$ is the spatial period of the array, $\lambda_{J}$ is the Josephson penetration depth, $\omega_{R}$ is the dissipation frequency, $J_{c}^{(n)} / J_{c}$ is the ratio of the critical current $J_{c}^{(n)}$ in the $n$th junction and the average critical current $J_{c}$, and $j_{n}$ $=J_{n} / J_{c}$ is the normalized external current flowing through the $n$th junction. In the experiment, ${ }^{31}$ the discreteness parameter can be chosen arbitrarily, e.g., it can take on any value between 0.1 and 10 .

Let us first consider the case when all contacts are identical, $J_{c}^{(n)} / J_{c}=1$, while we can change the driving current $j_{n}$ through the junctions. The solution of Eq. (1) for plasma waves can be written in the form $\phi_{n}=\phi_{n}^{(0)}+\varphi_{n}$, with $\varphi_{n} \ll 1$, where

$$
-j_{n}=\frac{1}{a^{2}}\left(\phi_{n+1}^{(0)}+\phi_{n-1}^{(0)}-2 \phi_{n}^{(0)}\right)-\sin \phi_{n}^{(0)}
$$

and

$$
\frac{1}{\omega_{J}^{2}} \frac{d^{2} \varphi_{n}}{d t^{2}}+\frac{1}{\omega_{R}} \frac{d \varphi_{n}}{d t}+\left(1+\delta_{n}\right) \varphi_{n}=\frac{1}{a^{2}}\left(\varphi_{n+1}+\varphi_{n-1}-2 \varphi_{n}\right)
$$

with $\delta_{n}=\cos \phi_{n}^{(0)}-1$ which can be seen as a tunable (by changing $j_{n}$ ) "potential" for JPW. In this sense, Eq. (2) can be considered as a recipe to calculate the current distribution which has to be used to obtain a desirable set of $\phi_{n}^{(0)}$ and, thus, $\delta_{n}$. Instead of currents $j_{n}$, magnetic fields applied between adjacent Josephson junctions can also be used to produce a tunable potential for JPWs.
An alternative way to create a potential $\delta_{n}$ for JPWs would be the fabrication of a discrete transmission line with a desirable distribution of the parameters. For instance, the critical current of the $n$th junction can vary, taking the values $J_{c}^{(n)}=J_{c}\left(1+\delta_{n}\right)$ with the zero-averaged $\delta_{n}$, i.e., $\left\langle\delta_{n}\right\rangle=0$. Considering JPWs with $\phi_{n}=\varphi_{n} \ll 1$, we recover Eq. (3), but now with a fixed potential.

In practice, one can never change the critical current alone. For example, one can design the junction area to be different from junction to junction. This will give an extra fluctuating coefficient in front of the first term of Eq. (3). Taking into account this coefficient results in the renormalization of the fluctuating parameter $\delta_{n}$. The exact expression for $\delta_{n}$ depends on the design of the experiment.

\section{B. Mapping the Josephson chain onto a tight-binding model}

In order to map the problem of propagating JPWs in a transmission line [Fig. 1(a)], we need to neglect both energy dissipation and nonlinearity. The dissipation term in Eq. (3) can be safely omitted for low-enough temperatures, while the nonlinearity becomes important ${ }^{6}$ only for high-enough amplitudes of the plasma waves. As a result, the equation describing the propagation of linear JPWs in this discrete system can be rewritten as

$$
\varphi_{n+1}+\varphi_{n-1}+U_{n} \varphi_{n}=E \varphi_{n}
$$

with

$$
U_{n}=a^{2} \delta_{n} ; \quad E(\omega)=2-a^{2}\left(\frac{\omega^{2}}{\omega_{J}^{2}}-1\right) .
$$

Below we assume $U_{n} \ll 1$, which can be easily satisfied by an appropriate choice of $\delta_{n}$ and $a$.

Equation (4) is the same as in the tight-binding model proposed by Anderson ${ }^{11}$ for the description of quantum particle motion in discrete 1D uncorrelated site potentials $U_{n}$. For $U_{n}=0$ (i.e., at $\delta_{n}=0$ ) the particle is delocalized,

$$
\varphi_{n}=\varphi_{0} \exp (\text { iqn }), \quad E=2 \cos q .
$$

This corresponds to the known result ${ }^{31}$ that JPWs with the wave number $q$ and

$$
\frac{\omega^{2}}{\omega_{J}^{2}}=1+\frac{4}{a^{2}} \sin ^{2}(q / 2)
$$

can propagate in the transmission line. For periodic $U_{n}$ (i.e., periodic $\delta_{n}$ ), the amplitude in Eq. (6) slightly oscillates, $\varphi_{0}(n)=\varphi_{0}+\phi(n) ;|\phi(n)| \ll \varphi_{0}$, with the period of the potential $U_{n}$. For this case, the zone structure of $E$ (photonic crystal for the JPW spectrum (see, Ref. 4) or energy zone structure for quantum particles) occurs. For uncorrelated (white noise) random potentials $U_{n}$, the envelope curve $\varphi_{0}(n)$ decays exponentially due to localization. According to Thouless, ${ }^{12}$ the localization length $l_{0}^{\text {quant }}$ for quantum particles obeys the relation,

$$
l_{0}^{\text {quant }}(E)=8 d \sin ^{2} q(E) / U_{0}^{2},
$$

where $d$ is the spacing between the potential sites, $U_{0}^{2}$ $=\left\langle U_{n}^{2}\right\rangle$ is the variance of the random potential. For Josephson 
$1 \mathrm{D}$ arrays, the localization length $l_{0}^{\mathrm{JPW}}(\omega)=l_{0}^{\text {quant }}[E(\omega)]$ can be rewritten as

$$
l_{0}^{\mathrm{JPW}}(\omega)=\frac{8 d a^{2}}{U_{0}^{2}}\left(\frac{\omega^{2}}{\omega_{J}^{2}}-1\right)\left[1-\frac{a^{2}}{4}\left(\frac{\omega^{2}}{\omega_{J}^{2}}-1\right)\right] .
$$

Using this result, we can conclude that the transparency $T(\omega)$ of the transmission line is exponentially small, i.e.,

$$
T(\omega) \propto \exp \left[-\frac{L}{l_{0}^{\mathrm{PP}}(\omega)}\right],
$$

if the sample length $L$ exceeds the localization length $l_{0}^{\mathrm{JPW}}$ (see, e.g., Ref. 32). For frequencies close to $\omega_{J}$, localization occurs if the number $N$ of junctions in the array exceeds $2 / U_{0}^{2 / 3}$. Here we take into account that $q>q_{\min }=1 / N$. The transmission line becomes completely nontransparent (for any frequencies) if $N \gg 8 / U_{0}^{2}$.

\section{Transparency edge due to correlated disorder}

Correlations in the random potential introduce some degree of order and thus lead to an increase in the localization length. According to Refs. 14, 15, 25, and 26, the localization length $l^{\text {quant }}(E)$ for quantum particles is determined by the Fourier transform of the normalized correlator

$$
\xi(r)=\frac{\left\langle U_{n} U_{n+r}\right\rangle}{U_{0}^{2}},
$$

where $\langle\cdots\rangle$ denotes the average over the chain:

$$
\begin{gathered}
l^{\text {quant }}(E) \equiv l^{\text {quant }}[E, q(E)]=\frac{q_{0}^{\text {quant }}(E)}{\widetilde{\xi}(2 q)}, \\
\widetilde{\xi}(q)=1+2 \sum_{r=1}^{\infty} \xi(r) \cos (q r)
\end{gathered}
$$

with $q(E)$ defined by Eq. (6). For the white-noise potential, $\xi(r)=\delta_{0, r}$, and Eq. (12) gives $l^{\text {quant }}(E)=l_{0}^{\text {quant }}(E)$, i.e., we obtain the previous result Eq. (8). In the case of short-range correlations, the correlator $\xi(r)$ for several values of $r$ is different from zero and the Fourier component $\tilde{\xi}(q)$ can only vanish at some discrete energies, where $\widetilde{\xi}(q)=0$. Thus, shortrange correlations of random potentials can result in extended states ${ }^{22}$ at several isolated energies.

If the correlations are long-range [e.g., $\xi(r)$ decays as a power law], a continuum of extended states can appear in the energy spectrum, ${ }^{16,25,33}$ since $\widetilde{\xi}(q)$ can vanish in an interval of $q$. In other words, controllable long-range correlations facilitate the observation of the metal-insulator transition. ${ }^{30}$ Therefore, transparency windows can be observed in the transmission line, if one fabricates either a transmission line with a locally changeable external current and apply $j_{n}$ with desirable correlations, or a junction array with fixed appropriate correlations of $J_{c}^{n}$.

There exist several methods of generating partially random sequences with desirable correlation functions. For example, a random set of scattering centers, with continuously distributed parameters,${ }^{30}$ was used to manipulate microwave transmission spectra through a single-mode waveguide. However, this requires a precise control of the system parameters. Recently, it was shown ${ }^{34}$ that random sequences with any prescribed correlation function can be constructed using additive many-step Markov chains with long-range memory function. ${ }^{35}$ In this case, it is possible to use only two values for the random variable, which is much easier to control during the fabrication process. Thus, for example, we can consider arrays of junctions where the Josephson current takes only two values, $J_{c}^{(n)}=J_{c}(1 \pm \delta)$. Previous related work on acoustic wave propagation in semiconductor multilayers appears in Ref. 16.

Using our Eqs. (5)-(12), we now calculate the frequency dependence of the transmission coefficient

$$
T / T_{0}=T / T(\delta=0)=\exp \left[-L / l^{\mathrm{JPW}}(\omega)\right],
$$

normalized to the value of $T$ at zero fluctuations. A correlated random binary sequence (the concrete realization of binary sequences in Ref. 36 which were used when plotting Figs. 2-4) of $10^{5}$ symbols (either zero or one) was generated by means of the method described in Ref. 34 and the Appendix. The sizes of the samples (number of symbols) were chosen to get significant filtering effect which gradually decays for smaller samples.

The results of the calculations of $T(\omega) / T_{0}$ are presented for transmission lines in Figs. 2(a) and 3(a). The dashed blue curves correspond to uncorrelated disorder, which strongly suppresses the transmissivity for long-enough samples. The solid red curves describe the transmissivity of the samples with long-range correlated disorder having the correlation function shown in Figs. 2(b), 2(c), 3(b), and 3(c). The correlation function in Fig. 2 was chosen so that it suppresses the transmission of $\mathrm{THz}$ waves at the middle $\left(\omega / \omega_{J}\right)^{2} \approx 1$ $+2 / a^{2}$ of the spectrum located in

$$
1<\frac{\omega}{\omega_{J}}<\sqrt{1+\frac{4}{a^{2}}}
$$

while the transmission of $\mathrm{THz}$ waves with frequencies near the edge of the spectrum is suppressed for the correlations used here to obtain Fig. 3. Thus, it is clearly seen that introducing correlations allows to obtain transparency windows and frequency-selective filtering.

\section{THE TRANSPARENCY EDGE IN SUPERCONDUCTING MULTILAYERS}

Now we consider superconducting multilayers [Fig. 1(b)] with fluctuating Josephson current, $J_{c}^{(n)}=J_{c}\left(1+\delta_{n}\right)$, between the $n$th and $(n+1)$ th layers. This system is described by the coupled sine-Gordon equations ${ }^{37}$

$$
\left(1-\frac{\lambda_{a b}^{2}}{s^{2}} \Delta_{n}\right)\left\{\frac{\partial^{2} \varphi_{n}}{\partial t^{2}}+\omega_{J}^{2} \sin \left(\varphi_{n}\right)\right\}-\frac{c^{2}}{\varepsilon} \frac{\partial^{2} \varphi}{\partial x^{2}}=0,
$$

where $\lambda_{a b}$ is the in-plane London penetration depth, $s$ is the interlayer spacing, $\varepsilon$ is the interlayer dielectric constant, and the operator $\Delta_{n}$ is defined as $\Delta_{n} f=f_{n+1}-2 f_{n}+f_{n-1}$. Lineariz- 

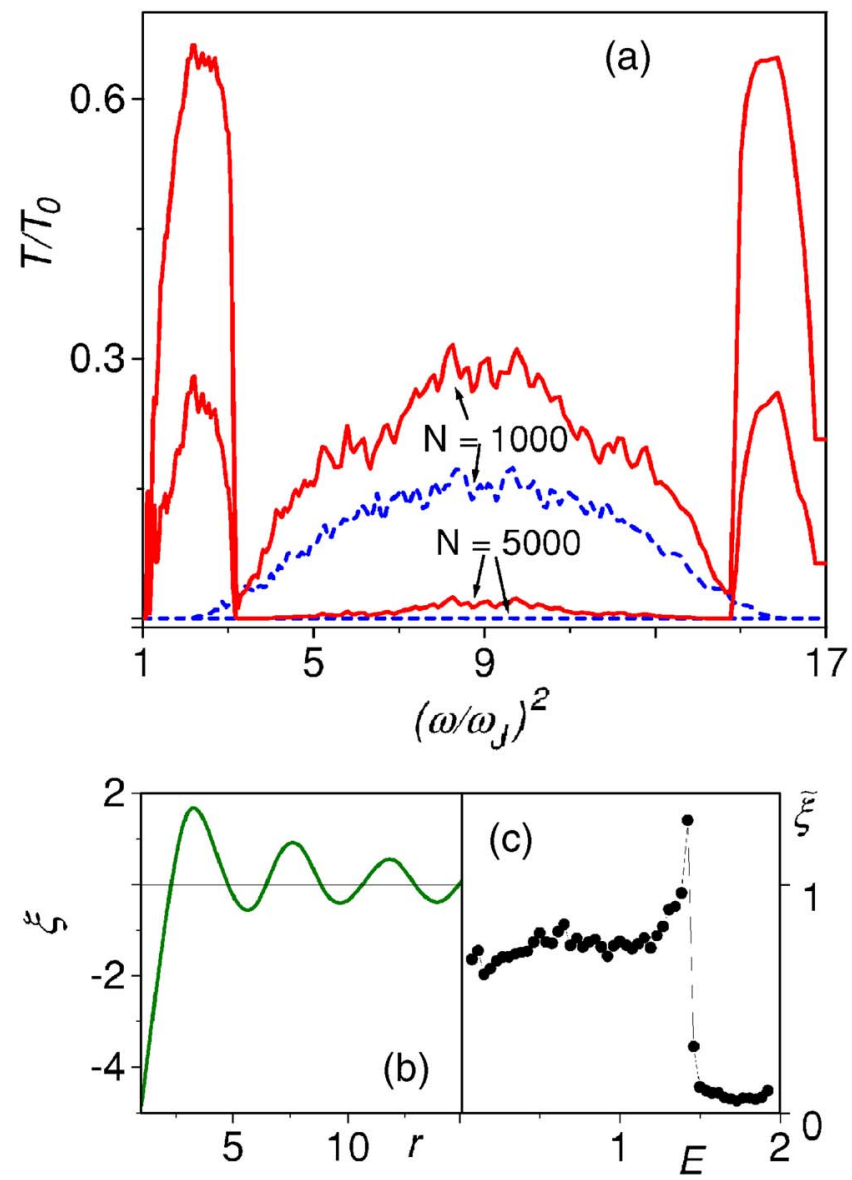

FIG. 2. (Color online) Controlling transparency windows in frequency-selective $\mathrm{THz}$ filters [Fig. 1(a)]. (a) The dimensionless transmission coefficient $T / T_{0}=T / T(\delta=0)=T(\omega) / T(\omega, \delta=0)$ versus dimensionless frequency $\omega / \omega_{J}$ for different lengths $N=L / d$ of Josephson chains [Fig. 1(a)]. Blue dashed lines correspond to $T(\omega)$ for uncorrelated disorder, while the red solid curves correspond to $T(\omega)$ with disorder having correlation functions which are shown in panels (b) and (c). Spatial correlations associated with dimensionless $\xi(r)$ (normalized by $a$ ), shown in panel (b), produce correlations in the frequency domain: shown in the Fourier transform $\widetilde{\xi}(E)$ with dimensionless $E$ [see panel (c)]. The parameters used here are: $a$ $=0.5$; dispersion $\propto U_{0}^{2}=10^{-2}$. It is clearly seen that varying the correlation function $\xi$ controls the transparency windows in $T(\omega)$.

ing this equation $\left[\sin \left(\varphi_{n}\right) \rightarrow \varphi_{n}\right]$, and considering a monochromatic THz wave with frequency $\omega$ and wave vector $\omega / c$ incident from the vacuum to the ab-plane of the sample, we obtain

$$
\varphi_{n}(x, t)=\tilde{\varphi}_{n} \exp \left(i \omega t-i \frac{\omega \sin \theta}{c} x\right),
$$

where $\theta$ is the incident angle of the $\mathrm{THz}$ waves [see Fig. 1(b)]. Here we assume that the in-plane component of the wave vector cannot change at the sample boundary. For $\tilde{\varphi}_{n}$ we derive Eq. (15) in the same form as Eq. (4) with

$$
E=2-\frac{s^{2}}{\lambda_{a b}^{2}}\left(\frac{\omega^{2} \sin ^{2} \theta}{\varepsilon\left(\omega^{2}-\omega_{J}^{2}\right)}-1\right),
$$

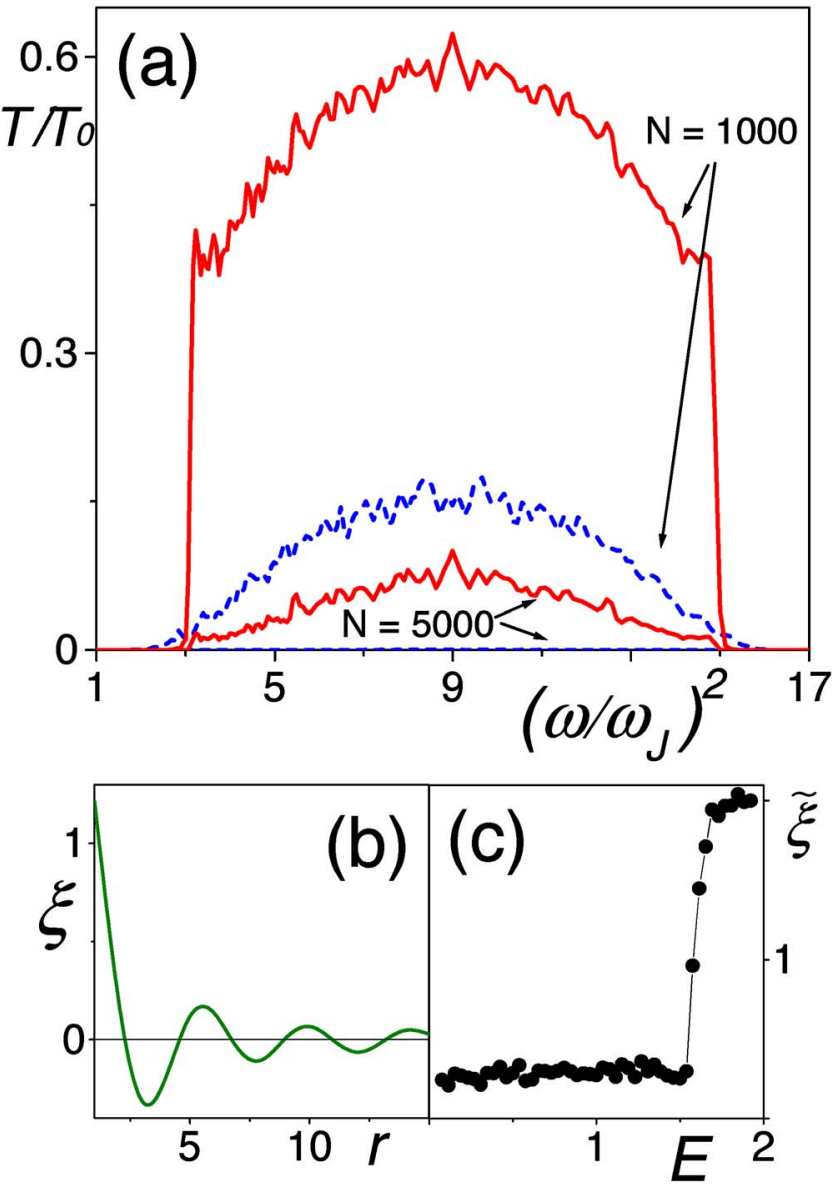

FIG. 3. (Color online) Controlling the transparency windows in frequency-selective $\mathrm{THz}$ filters [Fig. 1(a)]: Same as in Fig. 2, but with dimensionless correlation functions $\xi$ shown in (b) and (c). Comparing Figs. 2 and 3, it is clearly seen that varying the correlation function $\xi$ controls the transparency windows in $T(\omega)$. Notice the sharp change in the correlation function $\widetilde{\xi}(E)$ in both, Figs. 2(c) and 3(c).

$$
U_{n}=\frac{s^{2}}{\lambda_{a b}^{2}} \frac{\omega^{2} \sin ^{2} \theta}{\varepsilon\left(\omega^{2}-\omega_{J}^{2}\right)^{2}} \cdot \delta_{n} .
$$

In order to obtain the expression (18) for random potentials, we use the relation

$$
\left(\omega_{J}^{(n)}\right)^{2}=\frac{8 \pi e s J_{c}^{(n)}}{\hbar \epsilon}=\omega_{J}^{2}\left(1+\delta_{n}\right)
$$

with $\omega_{J}^{2}=8 \pi e s J_{c} / \hbar \epsilon$.

Thus, we show that the coupled sine-Gordon model can be also mapped to the tight-binding model (4). For samples with correlated disorder, one can observe the transmissivity window with respect to either the incident angle $\theta$ or frequency $\omega$ offering the possibility of filtering sub-THz and $\mathrm{THz}$ radiation with respect to frequency and/or direction of the wave propagation determined by the angle $\theta$. For instance, one can consider the incident angle $\theta$ as a control parameter for the frequency windows in $T(\omega)$, i.e., for the transparency edge in superconducting multilayers. For a layered sample with correlated disorder having the correlation 


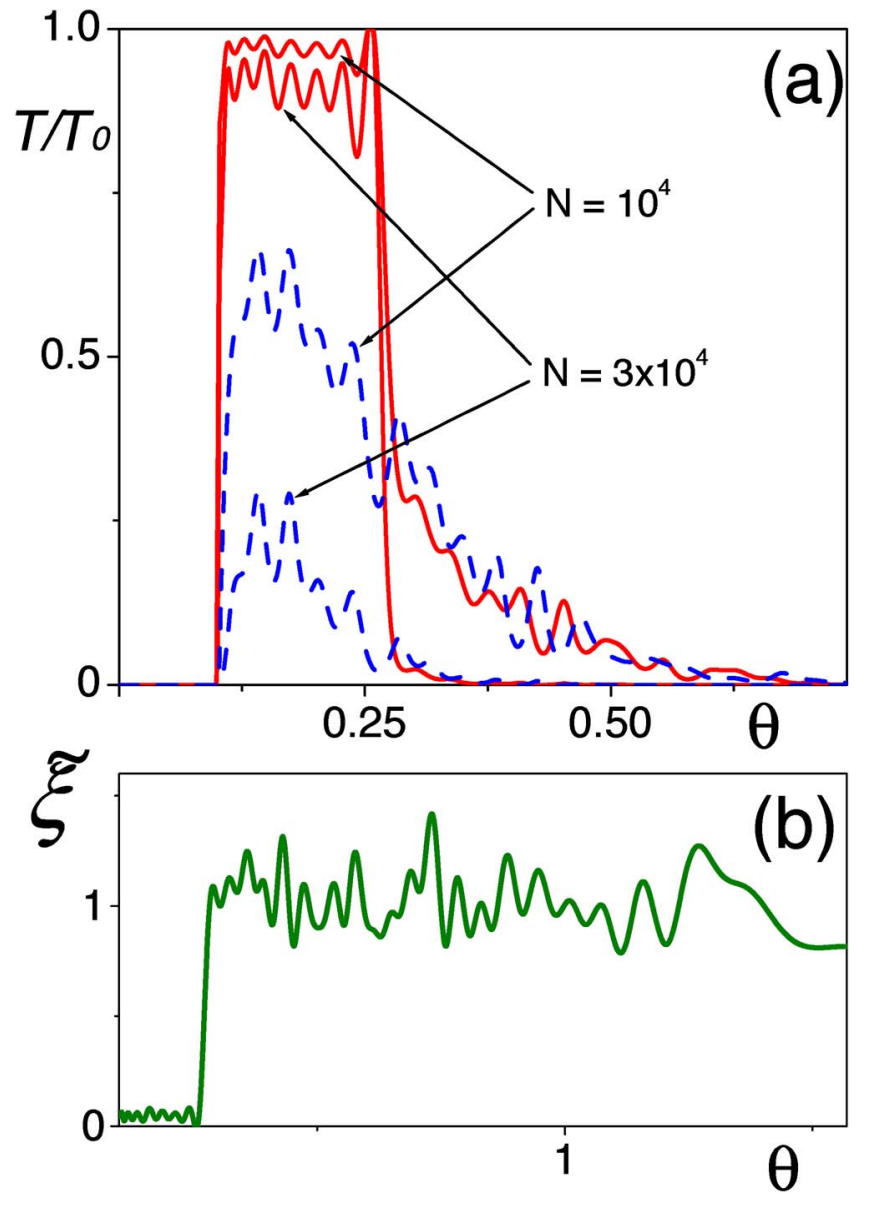

FIG. 4. (Color online) Controlling transparency windows in angle-selective THz filters. (a) The dimensionless transmission coefficient $T / T(\delta=0)$ versus the incident angle $\theta$ of $\mathrm{THz}$ radiation [see Fig. 1(b)] for different thickness $N=L / d$ of artificial multilayers [Fig. 1(b)]. Blue dashed lines correspond to $T(\theta)$ for uncorrelated disorder in the Josephson critical currents between layers, while the red solid curves correspond to $T(\theta)$ with correlated disorder. Correlations are described by the dimensionless angularcorrelation function $\tilde{\xi}(\theta)$ shown in panel (b). The parameters used here are $\lambda_{a b} / \lambda_{c}=200, \delta=10^{-3},\left(\omega^{2}-\omega_{J}^{2}\right) / \omega_{J}^{2}=10^{-3}$, and $\varepsilon=10$.

function shown in Fig. 4(b), the transparency versus the incident angle is shown in Fig. 4(a). The filtering of THz radiation with respect to the incident angle is clearly seen.

\section{CONCLUSIONS}

We show that wave localization occurs for JPWs propagating in either disordered 1D Josephson arrays (1D Josephson transmission lines) or artificial supercoducting multilayers. For samples with correlated disorder, transparency windows can be observed when varying the frequency or the angle of the incident electromagnetic waves. This transparency edge can be changed at will by an appropriate choice of the correlation function, which could be useful for $\mathrm{THz}$ devices, including frequency-selective or direction-selective filters. Also, the possibility to control the nonlinearity and dissipation in Josephson chains and arrays opens an avenue for experimental studies of these effects on localization. For example, it would be of interest to construct a transmission line with a quasiperiodic sequence of junctions where a singular continuous spectrum of states is realized. In this case, the power-law decrease of the transmission coefficient with an increase of the sample length should be observed. ${ }^{16,38}$ Moreover, experimental and theoretical studies of JPW propagation in 2D Josephson arrays could be a useful tool to study wave localization in 2D structures.

\section{ACKNOWLEDGMENTS}

We gratefully acknowledge conversations with A. Ustinov, and partial support from the National Security Agency (NSA), Laboratory Physical Science (LPS), Army Research Office (ARO), National Science Foundation (NSF) Grant No. EIA-0130383, JSPS-RFBR 06-02-91200, and Core-toCore (CTC) program supported by Japan Society for Promotion of Science (JSPS). S.S. acknowledges support from the Ministry of Science, Culture and Sport of Japan via the Grant-in Aid No. 18740224, the EPSRC Advanced Research Fellowship No. EP/D072581/1, and ESF networkprogramme "Arrays of Quantum Dots and Josephson Junctions".

\section{APPENDIX: GENERATION OF THE CORRELATED RANDOM BINARY SEQUENCE}

Here we present a short description of the method ${ }^{34}$ to generate binary sequences of zeros and ones with desirable correlation properties. Consider a homogeneous binary sequence, $\delta_{i}= \pm \delta$, of symbols for $-\mathcal{N} \leq i \leq \mathcal{N}$. To construct an $N$-step Markov chain we have to introduce the conditional probability $P\left(\delta_{i} \mid \delta_{i-N}, \delta_{i-N+1}, \ldots, \delta_{i-1}\right)$ for the $i$ th symbol $\delta_{i}$ to take the value $\delta_{i}=+\delta$ if the $N$ symbols before the $i$ th one have taken values $\delta_{i-N}, \delta_{i-N+1}, \ldots, \delta_{i-1}$ (i.e., if the $i$ th symbol occurring after the $N$-length-word $T_{N, i}$, where $T_{N, i}$ stands for the sequence of symbols $\delta_{i-N}, \delta_{i-N+1}, \ldots, \delta_{i-1}$ ) (another study of $N$-step Markov chains was done in Ref. 16). The additive Markov chain is characterized by the conditional probability function of the form

$$
P\left(\delta_{i}=\delta \mid T_{N, i}\right)=\frac{1}{2}+\sum_{r=1}^{N} F(r) \delta_{i-r},
$$

where the function $F(r)$ is the memory function. The memory function describes how strong a previous symbol $\delta_{i-r}$ affects the generated one, $\delta_{i}$. There is a relation, see Ref. 34, between the memory function $F(r)$ and the correlation function $\xi(r)$ of the Markov chain,

$$
\xi(r)=2 \delta \sum_{r^{\prime}=1}^{N} F\left(r^{\prime}\right) \xi\left(r-r^{\prime}\right), \quad r \geq 1 .
$$

This equation allows to obtain the memory function $F(r)$ and to generate a realization of Markov chain for any chosen (prescribed) correlation function $\xi(r)$. First, we choose a random set of initial symbols $\delta_{1}, \delta_{2}, \ldots, \delta_{N}$ with probability $1 / 2$ to take one of two values, $\delta_{i}= \pm \delta$. Using these symbols and 
the memory function $F(r)$, we calculate the conditional probability function, Eq. (20). When generating the $(N+1)$ th symbol, we assume the probability $p=P\left(\delta_{N+1}=\delta \mid T_{N, N+1}\right)$ of $\delta_{N+1}$ to take the value $+\delta$. Namely, we generate a random number $x$ homogeneously distributed within the interval $(0,1)$. If $x \in(0, p)$, we assign the symbol $\delta_{N+1}=+\delta$; else $\delta_{N+1}=-\delta$. At the next $(N+i)$ steps we employ the same pro- cedure using the word $\delta_{i}, \delta_{i+1}, \ldots, \delta_{i+N-1}$ as an input when calculating the conditional probability function. After the chain is constructed, the initial nonstationary part of the chain (of length $\geq N$ ) should be removed. Examples of such sequences are shown in Ref. 36. The symbols "zero" correspond to junctions with $J_{c}^{(n)}=J_{c}(1-\delta)$, while the symbols "one" correspond to junctions with $J_{c}^{(n)}=J_{c}(1+\delta)$.
${ }^{1}$ See, e.g., the special issue of Philos. Trans. R. Soc. London, 362, No 1815 (2004); B. Ferguson and X.-C. Zhang, Nat. Mater. 1, 26 (2002).

${ }^{2}$ R. Kleiner, F. Steinmeyer, G. Kunkel, and P. Müller, Phys. Rev. Lett. 68, 2394 (1992); G. Hechtfischer, R. Kleiner, A. V. Ustinov, and P. Müller, ibid. 79, 1365 (1997); E. Goldobin, A. Wallraff, N. Thyssen, and A. V. Ustinov, Phys. Rev. B 57, 130 (1998); J. Zitzmann, A. V. Ustinov, M. Levitchev, and S. Sakai, ibid. 66, 064527 (2002); M. Tachiki, M. Iizuka, K. Minami, S. Tejima, and H. Nakamura, ibid. 71, 134515 (2005).

${ }^{3}$ S. Savel'ev, V. Yampol'skii, A. Rakhmanov, and F. Nori, Phys. Rev. B 72, 144515 (2005); Physica C 437-438, 281 (2006); 445-448, 175 (2006).

${ }^{4}$ S. Savel'ev, A. L. Rakhmanov, and F. Nori, Phys. Rev. Lett. 94, 157004 (2005); Physica C 445-448, 180 (2006); Phys. Rev. B 74, 184512 (2006).

${ }^{5}$ S. Savel'ev, V. Yampol'skii, and F. Nori, Phys. Rev. Lett. 95, 187002 (2005); Physica C 445-448, 183 (2006).

${ }^{6}$ S. Savel'ev, A. L. Rakhmanov, V. A. Yampol'skii, and F. Nori, Nat. Phys. 2, 521 (2006); J. Orenstein, ibid. 2, 503 (2006).

${ }^{7}$ See, e.g., H. S. J. van der Zant, T. P. Orlando, S. Watanabe, and S. H. Strogatz, Phys. Rev. Lett. 74, 174 (1995); A. V. Ustinov, M. Cirillo, B. H. Larsen, V. A. Oboznov, P. Carelli, and G. Rotoli, Phys. Rev. B 51, 3081 (1995); J. Pfeiffer, M. Schuster, A. A. Abdumalikov, Jr., and A. V. Ustinov, Phys. Rev. Lett. 96, 034103 (2006); A. V. Ustinov, B. A. Malomed, and S. Sakai, Phys. Rev. B 57, 11691 (1998).

${ }^{8}$ J. Oppenlander, P. Caputo, Ch. Haussler, T. Trauble, J. Tomes, A. Friesch, and N. Schopohl, Appl. Phys. Lett. 83, 969 (2003).

${ }^{9}$ A. V. Ustinov and S. Sakai, Appl. Phys. Lett. 73, 686 (1998).

${ }^{10}$ Anderson Localization and Its Ramifications: Disorder, Phase Coherence, and Electron Correlations (Lecture Notes in Physics) edited by T. Brandes and S. Kettemann (Springer, Berlin, 2003); N. E. Hurt, Mathematical Physics of Quantum Wires and Devices: From Spectral Resonances to Anderson Localization (Springer, Berlin, 2000).

${ }^{11}$ P. Anderson, Phys. Rev. 109, 1492 (1958).

${ }^{12}$ D. J. Thouless, J. Phys. C 5, 77 (1972).

${ }^{13}$ J. D. Joannopoulos, R. D. Meade, and J. N. Winn, Photonic Crystals (Princeton University Press, Princeton, NJ, 1995).

${ }^{14}$ M. Griniasty and S. Fishman, Phys. Rev. Lett. 60, 1334 (1988).

${ }^{15}$ S. DasSarma, S. He, and X. C. Xie, Phys. Rev. Lett. 61, 2144 (1988).

${ }^{16}$ N. Nishiguchi, S. I. Tamura, and F. Nori, Phys. Rev. B 48, 14426 (1993); 48, 2515 (1993); S. I. Tamura and F. Nori, ibid. 41,
7941 (1990); 40, 9790 (1989).

${ }^{17}$ Q. Niu and F. Nori, Phys. Rev. Lett. 57, 2057 (1986); Phys. Rev. B 39, 2134 (1989); 42, 10329 (1990); F. Nori and J. P. Rodriguez, ibid. 34, 2207 (1986).

${ }^{18}$ M. Kolár and F. Nori, Phys. Rev. B 42, 1062 (1990).

${ }^{19}$ V. Misko, S. Savel'ev, and F. Nori, Phys. Rev. Lett. 95, 177007 (2005); V. R. Misko, S. Savel'ev, and F. Nori, Phys. Rev. B 74, 024522 (2006); V. R. Misko, S. Savel'ev, and F. Nori, Physica C 437-438, 213 (2006).

${ }^{20}$ N. L. Frisch and S. P. Lloyd, Phys. Rev. 120, 1175 (1960).

${ }^{21}$ B. Halperin, Phys. Rev. 139, A104 (1965).

${ }^{22}$ D. H. Dunlap, H.-L. Wu, and P. W. Phillips, Phys. Rev. Lett. 65, 88 (1990); P. Phillips and H.-L. Wu, Science 252, 1805 (1991).

${ }^{23}$ J. C. Flores, J. Phys.: Condens. Matter 1, 8471 (1989).

${ }^{24}$ A. Bovier, J. Phys. A, 25, 1021 (1992).

${ }^{25}$ F. M. Izrailev and A. A. Krokhin, Phys. Rev. Lett. 82, 4062 (1999).

${ }^{26}$ J. M. Luck, Phys. Rev. B 39, 5834 (1989); M. Kolár, M. K. Ali, and F. Nori, ibid. 43, 1034 (1991).

${ }^{27}$ F. M. Izrailev and N. M Makarov, J. Phys. A 38, 10613 (2005).

${ }^{28}$ H. Susanto, E. Goldobin, D. Koelle, R. Kleiner, and S. A. van Gils, Phys. Rev. B 71, 174510 (2005).

${ }^{29}$ V. Bellani, E. Diez, R. Hey, L. Toni, L. Tarricone, G. B. Parravicini, F. Domínguez-Adame, and R. Gómez-Alcalá, Phys. Rev. Lett. 82, 2159 (1999).

${ }^{30}$ U. Kuhl, F. M. Izrailev, A. A. Krokhin, and H.-J. Stockmann, Appl. Phys. Lett. 77, 633 (2000).

${ }^{31}$ A. V. Ustinov, M. Cirillo, and B. A. Malomed, Phys. Rev. B 47, 8357 (1993).

${ }^{32}$ D. J. Thouless, Phys. Rep. 13, 93 (1974).

${ }^{33}$ E. L. Albuquerque, M. S. Vasconcelos, M. L. Lyra, and F. A. B. F. de Moura, Phys. Rev. E 71, 021910 (2005).

${ }^{34}$ S. S. Melnyk, O. V. Usatenko, V. A. Yampol'skii, and V. A. Golick, Phys. Rev. E 72, 026140 (2005); S. S. Melnyk, O. V. Usatenko, and V. A. Yampol'skii, Physica A 361, 405 (2006).

${ }^{35}$ O. V. Usatenko and V. A. Yampol'skii, Phys. Rev. Lett. 90, 110601 (2003).

${ }^{36}$ www.dml.riken.go.jp/THz/localization.

${ }^{37}$ S. Sakai, P. Bodin, N. F. Pedersen, J. Appl. Phys. 73, 2411 (1993); M. Tachiki and M. Machida, Physica C 341-348, 1493 (2000); S. N. Artemenko and S. V. Remizov, ibid. 362, 200 (2001); Ch. Helm and L. N. Bulaevskii, Phys. Rev. B 66, 094514 (2002).

${ }^{38}$ O. V. Usatenko, S. S. Melnik, V. A. Yampol'skii, M. Johansson, L. Kroon, and R. Riklund, cond-mat/0507332 (unpublished). 